\title{
Participación política en América latina: UN ANÁLISIS DESDE LA PERSPECTIVA DE GÉNERO*
}

\author{
Political Participation in Latin America: \\ An Analysis from a Gender Perspective
}

\section{MÓNICA PACHÓN}

Universidad de los Andes

\section{XIMENA PEÑA}

Universidad de los Andes

\section{MÓNICA WILLS*}

Fundación Ideas para la Paz

\begin{abstract}
RESUMEN
A pesar de los avances en equidad de género en América Latina, existe aún una notable brecha en términos de participación política ciudadana. La investigación en América Latina se ha concentrado en entender la política como ocupación, razón por la cual la relación de los ciudadanos con la política constituye aún un vacío teórico. Evaluamos los determinantes de la participación política en América Latina, analizando a hombres y mujeres por separado. La ocupación, el ingreso y el estado civil están correlacionados con la participación política femenina, mientras que la confianza en hombres líderes y vivir en una zona urbana resultan ser determinantes en la participación política masculina.
\end{abstract}

Palabras clave: Participación política, género, índice por componentes principales. Códigos JEL: C21, D72.

\begin{abstract}
Despite the progress made in terms of gender equity in Latin America, there is still a significant gender gap in political participation. The literature has focused in the understanding of politics as an occupation, side-stepping the relationship of common citizens with the political environment. The present paper evaluates the determinants of the political participation in Latin America, analyzing men and women separately. The results suggest that occupation, income and marital status are associated with female political participation while the trust in male leaders and living in an urban region determine male participation.
\end{abstract}

Key words: Political participation, gender, principal components analysis.

JEL Codes: C21, D72.

* Agradecemos los comentarios de Christian Jaramillo así como de los evaluadores anónimos, sin duda fueron útiles para revisar el texto y mejorar el análisis. 


\section{INTRODUCCIÓN}

El estudio de la participación política en América Latina desde una perspectiva de género se ha centrado en encontrar los diferentes factores que llevan, tanto a hombres como a mujeres, a ocupar cargos públicos principalmente electorales (Donoso \& Valdés, 2007: 16). Sin embargo, la participación política por definición no se limita a la política como ocupación. Si el fundamento principal de la democracia radica en una participación igualitaria y equitativa entre hombres y mujeres, ¿por qué no se han explorado las diferencias individuales de participación entre éstos? ${ }^{1}$

Las mujeres en América Latina son menos activas políticamente que los hombres. En este documento se adoptaron cuatro dimensiones de la participación política: votación en elecciones presidenciales, trabajar en una campaña electoral, participar en una manifestación y hacer parte de un partido político.

El Foro Económico Mundial calcula desde el 2006 el índice de brecha de género con el propósito de medir, a nivel de países, el estado de avance de la mujer en términos de participación política. Este índice está compuesto por cuatro indicadores: participación económica, educación, salud y participación política. Cada uno de estos, al igual que el índice, posee un rango de 0 a 1 , donde 0 es total desigualdad y 1 es total igualdad entre hombres y mujeres.

El Gráfico 1 muestra el comportamiento de los indicadores anteriormente enunciados, tanto para el promedio mundial como para la OECD y para América Latina. Como es posible observar, la región latinoamericana se encuentra bien posicionada respecto al resto del mundo, en cuanto a temas de igualdad en salud, participación económica y educación entre hombres y mujeres. En contraste, se observa un desempeño notoriamente bajo en cuanto a participación política. Este indicador se encuentra por debajo tanto del promedio mundial como del promedio de la OECD.

Es importante estudiar la participación política en América Latina para entender sus determinantes. El presente artículo es una exploración cuantitativa para encontrar los determinantes de la participación política de los individuos, a partir de la inclusión de interacciones entre las variables mencionadas y la creación de un indicador de participación política. Todo esto utilizando la base de datos LAPOP 2008 Latin American Public Opinion Project. ${ }^{2}$

Los aportes de este artículo son varios. Primero, se estudia la participación política en América Latina, distinta a la política como ocupación. Segundo, se incorporan de manera

1 “La democracia presupone una asociación genuina entre hombres y mujeres en una sociedad, en la que trabajan en equidad, complementándose y enriqueciéndose de sus diferencias", Declaración Universal de la Democracia, 1997.

"The achievement of democracy presupposes a genuine partnership between men and women in the conduct of the affairs of society in which they work in equality and complementarity, drawing mutual enrichment from their differences", Universal Declaration of Democracy 1997.

2 Latin American Public Opinion Project (2008) del Barómetro Americano de la Universidad Vanderbildt en Nashville (Tennessee- Estados Unidos). 
Gráfico 1: Índice de brecha de género 2009

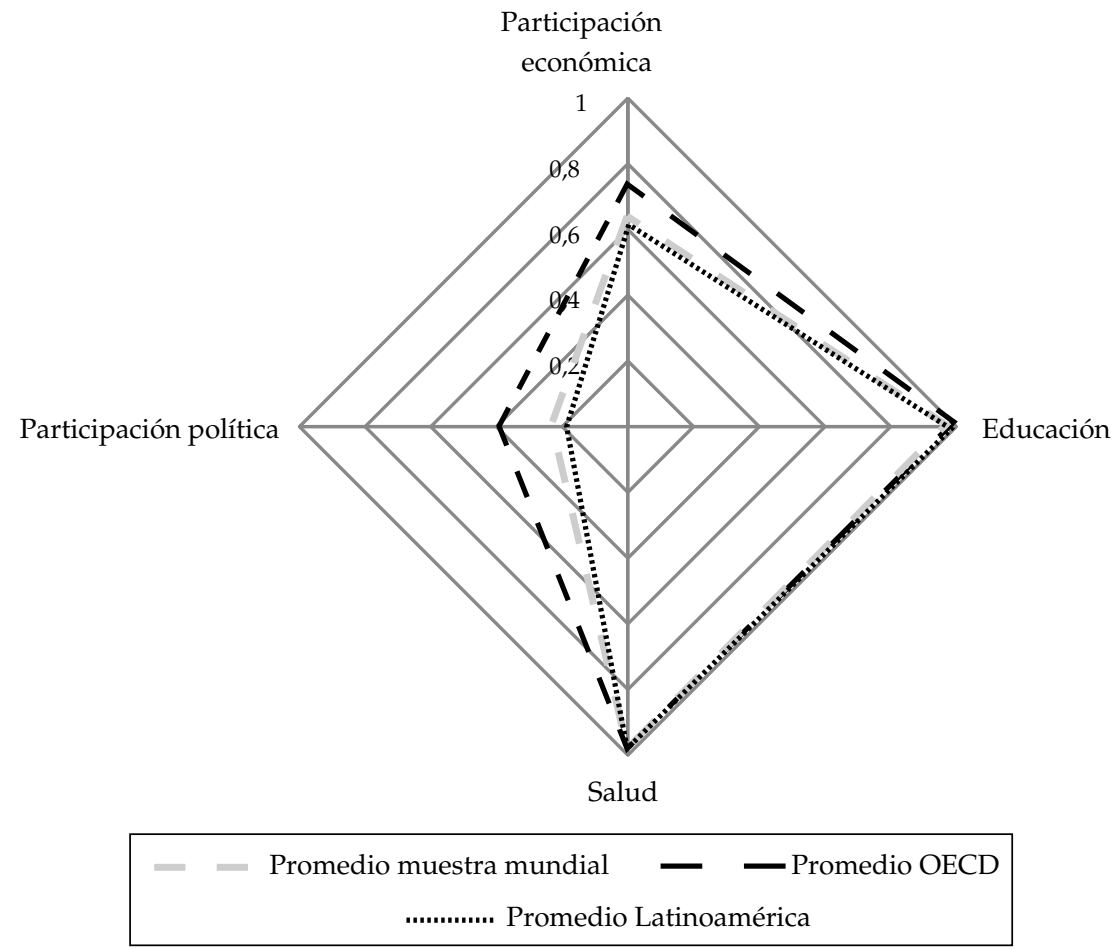

Fuente: Cálculos propios a partir del Foro Económico Mundial (2009).

simultánea las variables que la teoría ha resaltado, a saber: la ideología implícita y explícita, la confianza en hombres como líderes políticos, la percepción de discriminación, la ocupación y el tipo de ocupación. Exploramos si estas dimensiones explican las diferencias en participación política entre hombres y mujeres en América Latina.

En la siguiente sección se presenta un resumen de la literatura respecto al tema de participación política diferenciando por género. En la tercera sección se discute el modelo teórico que sirve como sustento a la estimación. En la cuarta sección se realiza una breve descripción de los datos que se utilizarán y se presentan las estadísticas descriptivas correspondientes. En el quinto apartado se realizan las estimaciones pertinentes y finalmente, en una sexta sección se exponen las conclusiones de la investigación.

\section{CONTEXTO DE LA INVESTIGACIÓN}

\subsection{El caso latinoamericano}

La participación política de las mujeres latinoamericanas podría resumirse por un lado, como una lucha constante por la ampliación del concepto de ciudadanía y por 
otro lado, como un proceso de construcción de un espacio de participación válido en la esfera pública. Las mujeres han ocupado el rol reproductivo al interior del hogar en la historia latinoamericana. Hasta principios del siglo pasado se encontraban confinadas a la esfera privada y excluidas casi por completo de la esfera pública (Llanos \& Sample, 2008: 11-15). Este hecho se fue modificando a lo largo del siglo XX, que puede ser considerado como el siglo de la transformación de la noción de género en Latinoamérica (Wills, 2005: 1).

La literatura de la historia de la participación femenina en América Latina hace referencia a dos momentos claves, que evidencian el esfuerzo, la organización y movilización de las mujeres en su paso a la política (Donoso \& Valdés, 2007: 16). El primer momento tuvo lugar en la primera mitad del siglo $X X{ }^{3}$ cuando las mujeres empezaron a incursionar en "el mundo de la cultura, la defensa de sus derechos sociales y laborales, la participación en organizaciones filantrópicas de beneficencia, en la organización y movilización de las primeras manifestaciones feministas y, sobre todo, en la lucha por los derechos civiles y ciudadanos representados en el sufragio" (Donoso \& Valdés, 2007: 16). Los resultados de esta lucha se materializaron con la obtención del sufragio femenino, siendo Ecuador el primero en 1929 y Paraguay el último en 1961 (FLACSO, 2006).

A partir de la década de los noventa se establecen a lo largo de América Latina diferentes leyes de cuotas, siendo pionero Argentina. Actualmente 10 países latinoamericanos poseen leyes de cuotas para candidaturas parlamentarias de alrededor del 20\% y el $40 \%$ (Peschard, 2003: 176). Por su parte, Colombia ha incorporado una cuota del 30\% para altos cargos ministeriales del Ejecutivo. Sin embargo, a pesar que los sistemas de cuotas han implicado una mayor vinculación de las mujeres a los cargos de decisión, resulta aún una medida insuficiente, como puede comprobarse con los resultados de la Tabla 1. Es notable que, a pesar que la participación de las mujeres ha aumentado, la representación actual no cumple aún la cuota para la mayoría de países latinoamericanos.

Tanto la elección por voto popular de mujeres a la presidencia de cinco países latinoamericanos, ${ }^{4}$ como el aumento en la participación de las mujeres en la rama legislativa $-150 \%$ entre el 2000 y el 2006 (Cordell, 2010)- evidencian el progreso en materia de equidad de género en el ejercicio de la política. Sin embargo, la participación ciudadana de las mujeres latinoamericanas sigue siendo inferior a la de los hombres (Donoso \& Valdés, 2007: 2).

3 Cabe aclarar que la contrucción de un concepto de ciudadanía con consideraciones de género se dio de manera contextual a cada pais en cada momento. Sin embargo es posible rescatar patrones que siguieron los paises latinoamericanos, como concecuencia de las similitudes en su composición social y cultural.

4 Violeta Barrios de Chamorro en 1990 en Nicaragua; Michelle Bachelet en 2006 en Chile; Mireya Moscoso en 1999 en Panamá; Cristina Fernández en 2007 en Argentina y Laura Chinchilla en 2010 en Costa Rica. 
Tabla 1: Composición por género de las Cámaras y Senados en las diferentes áreas de la región

\begin{tabular}{lcccc}
\hline \multicolumn{1}{c}{ País } & Año & $\begin{array}{c}\text { Cuota } \\
(\%)\end{array}$ & $\begin{array}{c}\text { Representación } \\
\text { antes de la ley }(\%)\end{array}$ & $\begin{array}{c}\text { Representación } \\
\text { actual }(\%)\end{array}$ \\
\hline Argentina & 1991 & 30 & 6 & 38,3 \\
Bolivia & 1997 & 30 & 11 & 16,9 \\
Brasil & 1997 & 30 & 7 & 8,8 \\
Costa Rica & 1996 & 40 & 14 & 36,8 \\
Ecuador & 1997 & 50 & 4 & 26 \\
Honduras & 2000 & 30 & 9,4 & 23,4 \\
México & 1996 & 30 & 17 & 22,6 \\
Panamá & 1997 & 30 & 8 & 15,3 \\
Paraguay & 1996 & 20 & 3 & 10 \\
Perú & 1997 & 30 & 11 & 29,2 \\
Rep. Dominicana & 1997 & 33 & 12 & 19,7 \\
\hline
\end{tabular}

Fuente: Cálculos propios en base a Estadísticas para la Equidad de Género: Magnitudes y tendencia en América Latina (Milosavljevic, 2007) y El sistema de cuotas en América Latina: Informe Regional (Peschard, 2003).

\subsection{Factores que inciden en la participación política de las mujeres}

La discusión sobre la representación identitaria versus la representación substantiva para el caso de la mujer pone de manifiesto el hecho de que por sí sola la condición de ser mujer no significa automáticamente la representación de sus intereses. Por ejemplo, para el caso colombiano, Wills (2007) argumenta que la ausencia de una identidad de género entre las candidatas y las votantes implica que no exista una representación real de las mujeres. En otras palabras, una mayor inclusión de las mujeres en los cargos de toma de decisiones no está relacionada con una mayor participación por parte de las electoras, debido a que no existe una identificación entre unas y otras. Por otro lado, Cole et al. (1998: 363) demuestran que las mujeres activistas se diferenciaban de las no activistas en que tienen una mayor conciencia de género. Es decir, se sentían mejor representadas por mujeres. La intuición tras esta afirmación radica en que las mujeres que declaran tener una mayor identidad de género tienden a estar vinculadas a grupos de mujeres, los cuales promueven el activismo politico de sus miembros.

Tanto Cole et al. (1998: 361), como Iversen y Rosenbluth (2006: 12), encuentran que las mujeres que tienen una ideología más de izquierda tienden a ser más activas políticamente. Por su parte, Iversen y Rosenbluth (2006: 14) hallan que los partidos de izquierda tienden a preocuparse más por el rol de la mujer en la política, y por una mayor visibilización de éstas en la esfera pública. Por lo tanto, las mujeres tienden a preferir candidatos que apoyan este tipo de iniciativas y participan más cuando siguen esta ideología. Por otro lado Cole et al. (1999: 352) encuentran que los partidos de izquierda tienden a favorecer 
políticas más acordes con los intereses de las mujeres (subsidios según número de hijos, proteccionismo estatal, a manera de seguro contra el divorcio), y por tanto, las mujeres con esta ideología política tienden a ser más activas políticamente.

Además de la ideología, la percepción de discriminación es también un factor importante para explicar el mayor involucramiento de las mujeres en la política (Lehman et al., 1999: 44). Sanbonmatsu (2003: 21) tiene resultados semejantes al encontrar que las mujeres que percibían discriminación hacia las mujeres entre los candidatos postulados en elecciones tendían a participar más. Ambas investigaciones justifican esta relación, en base a los incentivos generados por la percepción de discriminación. En otras palabras, la percepción de discriminación implica sentirse relegada de distintas áreas (esfera pública, laboral), y por tanto genera incentivos hacia el activismo, en la medida en que este constituye el ingreso a la esfera pública.

Otro de los factores que han demostrado ser importantes en explicar la mayor o menor participación de las mujeres es su ocupación. Tras un exhaustivo análisis de la división sexual del trabajo (incluyendo el trabajo doméstico), Iversen y Rosenbluth (2006: 12) encuentran que el pertenecer a una estructura jerárquica en el ámbito privado se refleja en una mayor participación en el ámbito público. Como ejemplo los autores proponen que tener subalternos o tomar decisiones empresariales fomenta la actitud participativa. Por su parte, Lehman et al. (1999: 36) encuentran que la existencia de responsabilidades familiares (tener hijos) implica que las mujeres no participen y se dediquen al hogar. De igual forma, los autores encuentran que las restricciones económicas operan en igual sentido, tanto para los hombres como para las mujeres, sobre la participación. ${ }^{5}$ En contraste, las restricciones familiares operan en sentidos opuestos, lo cual demuestra la importancia de entender el ámbito privado, para poder explicar la participación pública (bien sea laboral o política) de los individuos.

\section{LOS DATOS}

En este artículo usamos la base de datos de LAPOP 2008 -Latin American Public Opinion Project-, que es una encuesta de opinión pública que realiza el Barómetro Americano de la Universidad Vanderbilt en Nashville en cortes transversales repetidos anuales desde el 2001. La muestra consta de 25.421 observaciones, con aproximadamente 1.400 individuos por país, siendo estos representativos de cada uno de los países. Se tienen datos para Colombia, Bolivia, Ecuador, Perú, Venezuela, Costa Rica, El Salvador, Guatemala, Honduras, México, Nicaragua, Panamá, Paraguay, Uruguay, Brasil y República Dominicana. Dado que algunas de las preguntas no están en toda la muestra, la que se utiliza para los modelos se reduce alrededor de 15.000 observaciones.

5 Otro determinante importante de la participación de las mujeres se encontró en el tipo de trabajo. Es decir, se obtuvo que mujeres con trabajo de tiempo completo tienden a ser más activas políticamente, que mujeres con trabajos parciales (medio tiempo) o por horas. 
Esto nos permite abordar la participación y el activismo político desde la perspectiva del individualismo metodológico. Coincidimos con Iversen y Rosenbluth (2006: 2), quienes argumentan que el hogar como objeto de estudio discrimina e invisibiliza las negociaciones que se dan al interior del mismo. Estas negociaciones de roles, según los autores, afectan de manera significativa la toma de decisiones de los individuos, y por ende su participación política.

Tabla 2: Muestra LAPOP - América Latina 2008

\begin{tabular}{|c|c|c|c|c|c|}
\hline & Hombre & Mujer & \multirow{2}{*}{ Total } & \multirow{3}{*}{ T-Value } \\
\hline & & \multirow{2}{*}{$\frac{49,1}{\text { Porcentaje }}$} & \multirow{2}{*}{$\begin{array}{c}50,8 \\
\text { Porcentaje }\end{array}$} & & \\
\hline & & & & Porcentaje & \\
\hline \multirow[t]{4}{*}{ Lugar que habita } & Ciudad capital & 20,96 & 22,02 & 21,50 & $-2,64^{* * *}$ \\
\hline & Ciudad grande & 15,00 & 15,20 & 15,11 & \\
\hline & Ciudad mediana-pequeña & 30,19 & 30,59 & 30,45 & \\
\hline & Zona rural & 33,74 & 32,19 & 32,98 & \\
\hline \multirow[t]{6}{*}{ Educación ${ }^{6}$} & Nada & 3,95 & 5,55 & 4,76 & $-8,66^{* * *}$ \\
\hline & Algo de primaria & 18,32 & 20,59 & 19,47 & \\
\hline & Primaria completa & 14,65 & 15,29 & 14,97 & \\
\hline & Algo de secundaria & 29,34 & 28,56 & 28,94 & \\
\hline & Secundaria completa & 14,46 & 13,01 & 13,77 & \\
\hline & Terciaria & 19,29 & 16,93 & 18,09 & \\
\hline \multirow[t]{4}{*}{ Estado Civil } & Soltero & 38,17 & 32,82 & 35,42 & $22,13^{* * *}$ \\
\hline & Casado & 41,66 & 39,83 & 40,73 & \\
\hline & Unión libre & 18,23 & 20,84 & 19,56 & \\
\hline & Divorciado/Separado & 3,95 & 6,61 & 5,30 & \\
\hline
\end{tabular}

*** Significativo a $1 \%$. ** Significativo a $5 \%$. * Significativo a $10 \%$.

Fuente: Cálculos propios a partir de LAPOP, 2008.

La Tabla 2 presenta las estadísticas descriptivas en base a diferencias de género, mientras que la Tabla 3 contiene las diferencias en edad, educación y número de hijos por género. Del total de 25.421 en la muestra total para América Latina, el 50,8\% son mujeres. En promedio, los individuos tienen 39 años y alrededor de dos hijos. En cuanto al lugar que habitan, la mayor parte de la muestra se encuentra concentrada en áreas urbanas (67,20\%), compuestas por ciudades capitales $(20,96 \%)$, ciudades grandes $(15,11 \%)$ y ciudades de medianas a pequeñas $(30,45 \%)$. En promedio es posible comprobar que las mujeres y los 
Tabla 3: $\quad$ Promedio relevante de la muestra

\begin{tabular}{lcccc}
\hline & Hombre & Mujer & Total & T-Value \\
\hline Edad promedio & 38,58 & 38,92 & 38,74 & 1,12 \\
& $(16,43)$ & $(15,54)$ & $(15,98)$ & \\
Años de educación & 8,99 & 8,47 & 8,73 & $-8,83^{* * *}$ \\
& $(4,61)$ & $(6,68)$ & $(4,65)$ & \\
Número de hijos & 2,04 & 2,38 & 2,21 & $22,13^{* * *}$ \\
& $(1,08)$ & $(1,36)$ & $(1,24)$ & \\
\hline
\end{tabular}

Nota: Errores estándares en paréntesis. ${ }^{* * *}$ Significativo a 1\%. ** Significativo a 5\%. * Significativo a $10 \%$. Fuente: Cálculos propios a partir de LAPOP, 2008.

hombres reportan haber estudiado alrededor de 8 años. Sin embargo, las mujeres son menos educadas, habitan con más frecuencia en la capital, tienen más hijos, en menos probable que estén separadas o casadas y más probable que el concepto de "participación política" puede abarcar una gran variedad de dimensiones, que van desde ejercer el voto hasta ocupar un cargo público administrativo del Ejecutivo, como la presidencia de un país. Por un lado, es posible encontrar aproximaciones a la participación política, en las que se entiende la participación como el acto de votar (Iversen y Rosenbluth, 2006: 12; Inglehart \& Norris, 2003: 2). Sin embargo, esta aproximación resulta insuficiente, en la medida en que invisibiliza las demás dimensiones de la participación política. Por otro lado, la definición proporcionada por Conge (1988: 245) puede clasificarse como una maximalista:

"Participación política puede ser entonces definida como acciones individuales o colectivas, a nivel nacional o local, que apoyan o se oponen, de manera intencional o sin intención a estructuras estatales, autoridades y/o decisiones respecto a la distribución de los bienes públicos. Es necesario hacer énfasis en tres aspectos de esta definición. Primero la acción puede ser verbal o escrita. Segundo, puede ser violenta o pacífica. Tercero, puede ser de cualquier intensidad"(Conge, 1988: 245).

A pesar de la amplia gama de definiciones existentes entre la visión minimalista y maximalista de la participación política ciudadana, es posible encontrar cierto consenso en la literatura respecto a tres puntos claves. El primero hace alusión a la evidente relación entre la participación política y la democracia. Esto se debe a que la intervención de los ciudadanos en la toma de decisiones constituye la base sobre la que se encuentra sedimentada la democracia (Sabucedo, 1986: 59). El segundo punto radica en la relación de la participación política ciudadana y la distribución de bienes y servicios. Según Booth y Seligson (1978: 96) la participación política puede definirse como "acciones por parte de los individuos, que influencian o intentan influenciar la distribución de los bienes públicos" (Booth \& Seligson, 1978: 96). Finalmente, el tercer punto clave hace referencia a la clara diferenciación entre la participación política convencional y la no 
convencional. La participación política convencional se constituye por la participación fomentada y amparada por el Estado y la Constitución (Injuve, 2006). En especial, este tipo de participación hace referencia al proceso electoral (votar y trabajar en una campaña). La participación política no convencional va más allá de los mecanismos tradicionales e institucionales de participación, pues abarca acciones que van desde manifestaciones legales hasta huelgas ilegales y violencia (Injuve, 2006).

En nuestra investigación adoptaremos la definición de participación política amplia, en la cual se contemplan todas las acciones realizadas por los individuos, con el propósito de ejercer una acción política que tenga repercusiones en la sociedad que habitan sin que los individuos asuman la política como su principal ocupación. Es decir, se pretende explorar la relación que tiene el ciudadano común con la política.

Creamos un indicador de participación política que combina las variables de participación política disponibles en la encuesta LAPOP. La primera es voto por presidente, y constituye la acción política de participar a nivel electoral a partir de la elección de un candidato. La segunda es movilización, es decir, la participación en algún tipo de manifestación civil con objetivos políticos. La tercera, partido político, se refiere a pertenecer o estar vinculado a un partido político oficial dentro del país. La última es trabajar en una campaña electoral, que trabajar al lado de un candidato en época de elecciones, bien sea de forma voluntaria o remunerada.

Combinamos estas cuatro dimensiones en un indicador de participación política ${ }^{7}$ utilizando el análisis de componentes principales. La utilidad de este método radica en que al ponderar las variables independientes seleccionadas por los coeficientes provenientes de este análisis, se genera una combinación lineal de las mismas, que a su vez maximiza su varianza y elimina las potenciales covarianzas entre éstas. El indicador obtenido fue el siguiente:

$$
P P I_{i j}=0,5842 P_{i j}+0,5780 C_{i j}+0,4497 V_{i j}+0,3497 M_{i j}
$$

El indicador de componentes principales no posee un sustento teórico, en la medida en que la ponderación se otorga por medio de un proceso estadístico. Tras la construcción de la variable de participación política, se dividió la muestra en tres grupos por países según terciles de la participación política promedio. Los países con alta participación política (tercil superior) son Honduras, Nicaragua, Paraguay, República Dominicana y Uruguay. Los países con participación política media son Brasil, Bolivia, Panamá y Perú, y los de participación política baja son Colombia, Costa Rica, Ecuador, El Salvador, Guatemala y México.

7 Se realizaron análisis de robustez con dos maneras alternativas de calcular la participación política: un promedio aritmético simple de las categorías y uno ponderado, similar al propuesto por Burns, Schlozman y Verba (1999:46). Los pesos de este indicador serán asignados en base al nivel de activismo de la acción siguiendo la escala de Milbrath (1965). Los resultados presentados en el artículo son robustos a estas maneras alternativas de calcular la participación política. Los resultados presentados son robustos a usar un modelo logístico, en vez de MCO. 


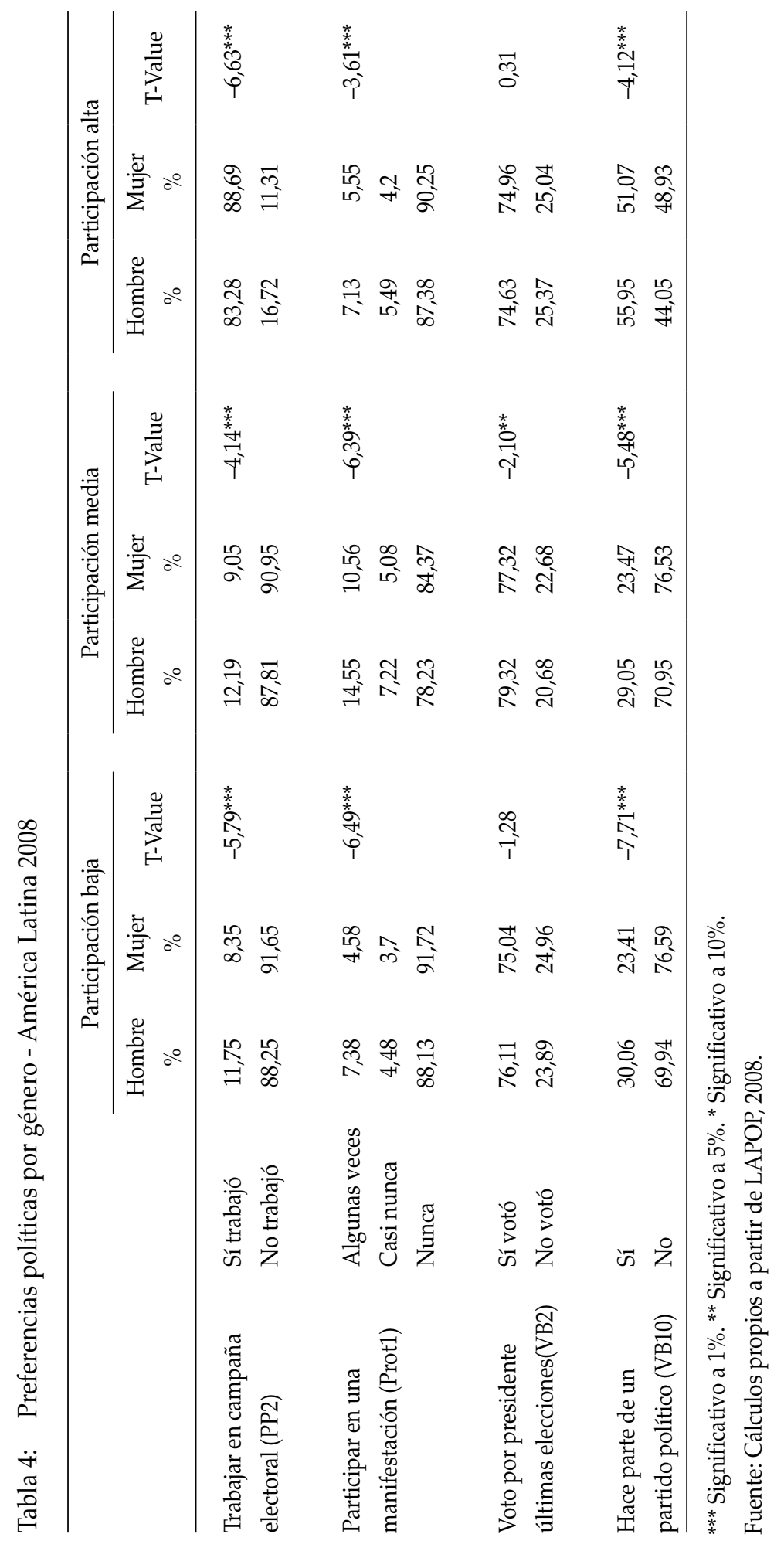


$\mathrm{Al}$ analizar el indicador, lo primero que se observa es que claramente la participación política de hombres y mujeres es bastante diferente. Las diferencias entre hombres y mujeres presentadas en los cuatro indicadores de participación política son en general estadísticamente significativas para los distintos grupos de participación política (Tabla 4).

Los hombres tienden a participar más en campañas políticas en los países con participación política baja o media. Sin embargo, las mujeres en países con alta participación participan con mayor frecuencia que los hombres en campañas políticas. Para todos los grupos de países, los hombres participan significativamente más en manifestaciones o pertenecen a un partido político. La diferencia menos estadísticamente relevante se encuentra en el voto por presidente en las últimas elecciones, comprobando el problema que constituiría incluirlo a manera de variable dependiente. En este caso, las diferencias de género son significativas únicamente para los países con participación intermedia. Si bien las diferencias de género no se hacen evidentes en la decisión electoral, las demás acciones políticas evidencian la desventaja de las mujeres frente a los hombres.

Ahora describamos las demás variables independientes. ${ }^{8}$ En la Tabla 5 es posible observar las diferencias en ocupación por género. Esta variable se recodificó de tal manera que fuera posible observar con mayor claridad las diferencias entre hombres y mujeres. Por lo tanto, se realizó una clasificación de los componentes en las tradicionales categorías de la ocupación: empleados, desempleados e inactivos, siendo estos últimos estudiantes, pensionados y amas de casa. Siguiendo a Iversen \& Rosenbluth (2006: 7), se clasificó cada una de las opciones del tipo de ocupación, en las siguientes tres categorías: Trabajo oficina, refiriéndose a tareas que requieren habilidades y que principalmente se desarrollan en una oficina. Trabajo manual, refiriéndose a actividades manuales y Servicios, refriéndose a los individuos que pertenecen al sector de servicios.

La variable tipo de ocupación no presenta una diferencia estadísticamente significativa. Sin embargo, los resultados de la ocupación sugieren una alta diferenciación de género. Con un $99 \%$ de significancia es posible comprobar que tan sólo el $36,14 \%$ de las mujeres se encuentran empleadas, en contraste con el $72,25 \%$ de los hombres. Con base en la teoría presentada se esperaría, entonces, que esta relación se traduzca en diferencias entre hombres y mujeres en participación política.

Por otro lado, la Tabla 6 presenta también las diferencias ideológicas entre hombres y mujeres en la región. Por medio de la encuesta de LAPOP 2008, se eligió como indicador de ideología la pregunta que pedía a los individuos ubicarse en una escala de 1 a 10 (siendo 1 extrema derecha y 10 extrema izquierda), según su ideología política. En otras palabras, la variable ideología explícita constituye un indicador de la ideología reportada por los encuestados, de manera que un valor más alto corresponde a una posición más hacia la izquierda.

Otra manera de capturar la ideología consta en preguntarle a los individuos acerca de percepciones y luego asociarlas al tipo de ideología que implican. En este artículo se crea

8 En el Anexo se presentan las estadísticas descriptivas de algunas de las variables independientes por grupos de países. 
Tabla 5: Estadísticas Descriptivas Variables Independientes

\begin{tabular}{|c|c|c|c|c|c|}
\hline Ocupación & & $\begin{array}{c}\text { Hombre } \\
\%\end{array}$ & $\begin{array}{c}\text { Mujer } \\
\%\end{array}$ & $\begin{array}{c}\text { Total } \\
\%\end{array}$ & T-Value \\
\hline \multirow[t]{3}{*}{ Ocupación } & Empleado & 72,26 & 36,14 & 53,69 & $17,81^{* * *}$ \\
\hline & Desempleado & 6,46 & 4,06 & 5,32 & \\
\hline & Inactivo & 21,09 & 59,80 & 40,99 & \\
\hline \multirow[t]{3}{*}{ Tipo de ocupación } & Trabajo oficina & 17,96 & 22,90 & 19,66 & 1,30 \\
\hline & Trabajo manual & 64,93 & 51,45 & 60,29 & \\
\hline & Servicios & 17,01 & 25,54 & 19,95 & \\
\hline \multicolumn{6}{|l|}{ Ideología } \\
\hline \multirow[t]{5}{*}{ Ideología explícita } & Izquierda Extrema & 10,79 & 10,16 & 10,99 & $2,79^{* * *}$ \\
\hline & Centro Izquierda & 15,43 & 14,94 & 15,1 & \\
\hline & Centro & 41,45 & 43,63 & 42,53 & \\
\hline & Centro Derecha & 17,59 & 18,17 & 17,52 & \\
\hline & Derecha Extrema & 13,94 & 13,36 & 13,61 & \\
\hline \multirow[t]{5}{*}{ Ideología implícita } & Izquierda Extrema & 14,15 & 16,98 & 15,56 & $-0,88$ \\
\hline & Centro Izquierda & 43,41 & 41,01 & 42,21 & \\
\hline & Centro & 31,49 & 32,28 & 31,88 & \\
\hline & Centro Derecha & 10,31 & 9,63 & 9,97 & \\
\hline & Derecha Extrema & 2,06 & 1,8 & 1,93 & \\
\hline \multicolumn{6}{|l|}{ Confianza en hombres } \\
\hline \multirow{4}{*}{$\begin{array}{l}\text { Los hombres son } \\
\text { mejores líderes } \\
\text { políticos }\end{array}$} & Muy de acuerdo & 11,38 & 7,03 & 9,18 & $25,35^{* * *}$ \\
\hline & De acuerdo & 24,91 & 15,55 & 20,18 & \\
\hline & En desacuerdo & 47,00 & 49,56 & 48,29 & \\
\hline & Muy en desacuerdo & 16,70 & 27,87 & 22,34 & \\
\hline \multicolumn{6}{|l|}{ Discriminación } \\
\hline \multirow[t]{2}{*}{ Oficinas del gobierno } & Sí & 12,54 & 11,13 & 11,83 & $2,23^{* * *}$ \\
\hline & No & 87,46 & 88,87 & 88,17 & \\
\hline \multirow[t]{2}{*}{ Buscando trabajo } & Sí & 10,93 & 9,95 & 10,42 & 0,67 \\
\hline & No & 87,09 & 87,83 & 87,48 & \\
\hline En reuniones o & Sí & 9,37 & 8,68 & 9,02 & 1,34 \\
\hline eventos sociales & No & 90,63 & 91,32 & 90,98 & \\
\hline \multirow[t]{2}{*}{ En lugares públicos } & Sí & 11,13 & 10,12 & 10,62 & 1,68 \\
\hline & No & 88,87 & 89,88 & 99,38 & \\
\hline
\end{tabular}

*** Significativo a $1 \%$. ${ }^{* *}$ Significativo a 5\%. * Significativo a 10\%.

Fuente: Cálculos de las autoras a partir de LAPOP, 2008. 


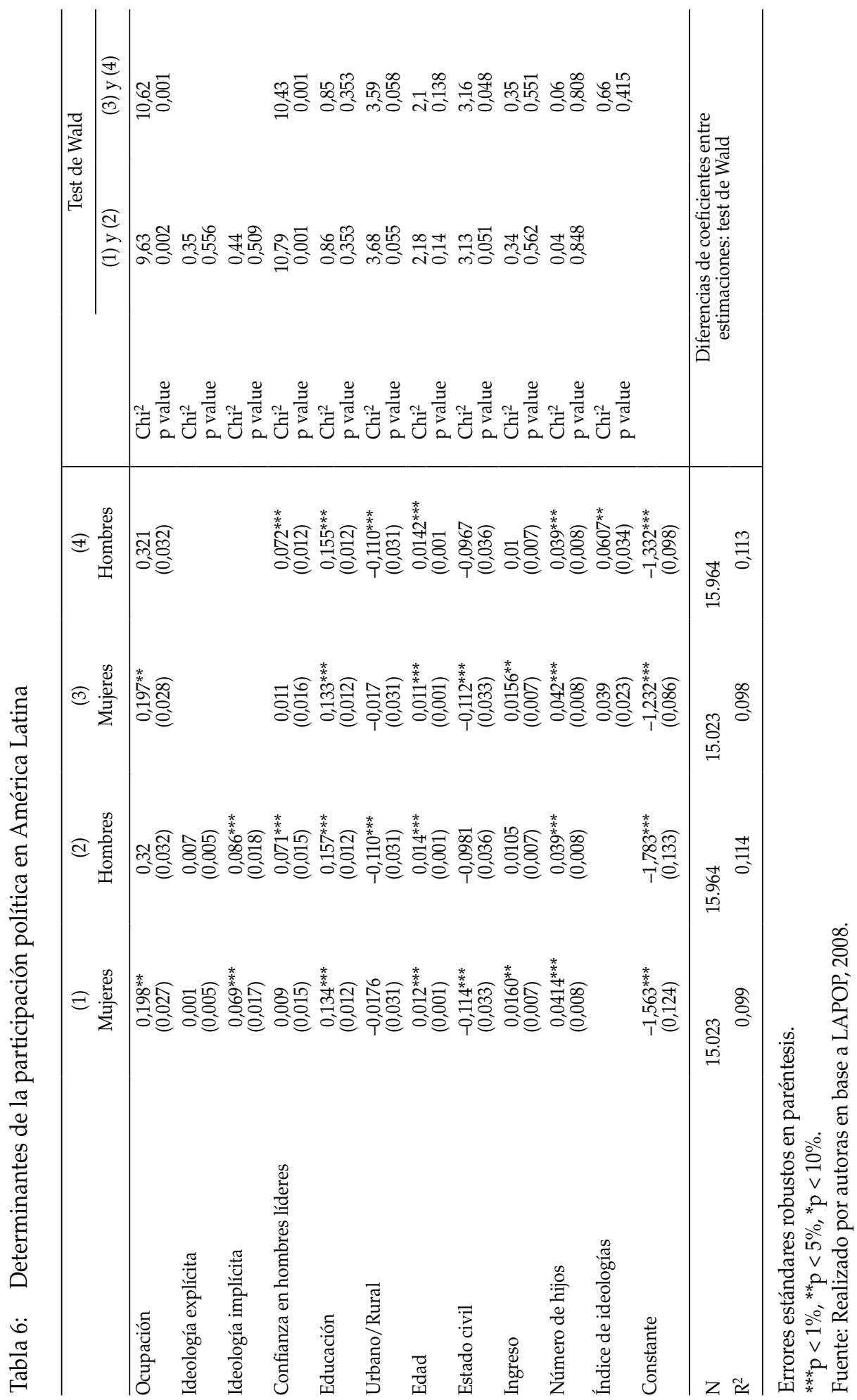


entonces un indicador de ideología implícita con base en la propuesta de Zeichmeister (2006). Para este fin, se escogieron las preguntas de la encuesta que coincidían con las definiciones de izquierda y derecha. El indicador de ideología implícita se construyó en base a la posición de los individuos respecto a diferentes afirmaciones en las que estos debían responder su nivel de acuerdo. ${ }^{9}$ A partir de las variables referenciadas en la misma se construyó un promedio aritmético como indicador de ideología implícita, donde un mayor valor de nuevo corresponde a una posición más hacia la izquierda.

Los resultados de ambos indicadores -ideología implícita y explícita- se presentan en la Tabla 5. A pesar de que las diferencias entre hombres y mujeres en cuanto a su ideología explícita resultan estadísticamente significativas, en términos de porcentajes no superan el 3\%: la distribución ideológica es similar para hombres y mujeres. Los hombres tienden a ser más de izquierda que las mujeres en su ideología explícita o autorreportada. Sin embargo, esto no genera diferencias en ideología implícita.

El presente trabajo no pretende probar diferencias ideológicas entre hombres y mujeres, sino el efecto que tengan éstas sobre su participación política. Para este punto, más que comparar las diferencias ideológicas entre hombres y mujeres, es prudente discutir las diferencias entre las variables construidas de ideología implícita y explícita. Como se muestra en la Tabla 5, los individuos de la muestra tienden a ser mucho más de izquierda de lo que reportan. Para la ideología explícita, un 26\% de los encuestados reporta ser de izquierda, mientras que la ideología implícita muestra que un 57,77\% coincide con los postulados de esta corriente. En cuanto a la derecha los resultados son opuestos. Un 21,03\% reporta seguir esta ideología, cuando en realidad solo el 11,9\% lo hace.

Incluimos además una variable de control que mide qué tanto las personas consideran que los hombres son mejores líderes políticos. Las estadísticas descriptivas muestran que los hombres tienden a estar más de acuerdo que las mujeres cuando se les pregunta si los hombres son mejores líderes políticos. Esto parece indicar que las mujeres son más abiertas a considerar a las mujeres como buenas líderes políticas que los hombres.

Finalmente, la Tabla 5 presenta también las diferencias en percepción de discriminación entre hombres y mujeres. Para definir la percepción de discriminación se utilizaron preguntas en las que se solicitaba a los individuos reportar si se habían sentido discriminados en distintas situaciones. Cabe aclarar que esta variable no implica discriminación per se, sino que da referencia de cómo se sienten los individuos en relación con diferentes entornos. De esta forma, es posible establecer si se han sentido discriminados en determinadas situaciones y si esto posteriormente ha afectado su participación política. En este caso, un menor porcentaje de mujeres reporta ser discriminada en cada uno de los ámbitos enunciados. Dado que los resultados sugieren que las cifras no están siendo

\footnotetext{
1. Impedir que minorías se impongan sobre la voluntad de la mayoría; 2 . El pueblo puede gobernar directamente y no a través de gobernantes electos; 3 . Los presidentes tienen que seguir la voluntad del pueblo, porque lo que el pueblo quiere es siempre lo correcto; 4 . El Estado, más que la empresa privada, debería ser el principal responsable de crear empleos; 5 . El Estado, más que los individuos, debería ser el principal responsable de asegurar el bienestar de la gente; 6 . El Estado, en lugar del sector privado, debería ser el dueño de las empresas e industrias más importantes del país.
} 
controladas por el tipo de discriminación (raza, etnia, género, nivel socioeconómico, etc.), no es posible estudiar empíricamente el efecto de la discriminación de género sobre la participación política.

\section{EL MODELO A ESTIMAR Y LOS RESULTADOS}

Siguiendo a Burns et al. (1999: 46) se estima un modelo que analiza la relación entre las variables anteriormente mencionadas y un indicador de participación política construido. La estimación se hace entonces, de manera separada para hombres y para mujeres, en base al siguiente modelo:

$$
P P I_{i j}=\alpha_{1}+\sum_{j=2}^{N}\left(\alpha_{1} \text { DummyPaís }\right)+\beta_{2} C H_{i j}+\beta_{3} I E_{i j}+\beta_{4} I I_{i j}+\beta_{5} O_{i j}+\beta_{6} X_{i j}+\varepsilon_{i j}
$$

Donde $P P I_{i j}$ es el índice de participación política para el individuo $i$ en el país $j$. En el lado derecho se encuentran la confianza en los hombres como líderes $\mathrm{ICH}_{i j}$, medida por una dummy donde 1 es preferir a una candidata y 0 a un candidato; la Ideología Explícita $I E_{i j}$ y la Ideología Implícita $I I_{i j}$ medidas por las escalas presentadas en la sección anterior y la Ocupación $O_{i j}$, medida por una dummy donde 1 es ocupado y 0 es inactivo. Las variables sociodemográficas, $X_{i j}$, son el estado civil, medido por una dummy de divorcio, ${ }^{10}$ la edad, la educación medida en años aprobados, el número de hijos, el ingreso, la raza y una variable dicótoma que toma el valor de 1 si la persona vive en una zona urbana. Incluimos también una constante y efectos fijos por país.

La Tabla 6 presenta la estimación de los determinantes de la participación política en América Latina, medida a través del indicador generado por componentes principales; el método de estimación es Mínimos Cuadrados Ordinarios (MCO). ${ }^{11}$ Separamos la muestra entre hombres y mujeres para determinar si hay diferencias estadísticamente significativas en los determinantes de la participación política entre géneros, usamos el Test de Wald.

La participación aumenta con la edad de las personas, los años educativos y el número de niños. Las personas con mayor nivel educativo suelen entender mejor las repercusiones de su participación política, así como suelen mostrar mayor interés por la misma. Por parte de la edad, se supone que en la medida en que las personas se independizan, trabajan y deben cumplir con ciertas responsabilidades con el Estado (impuestos), su interés por la política incrementa, al igual que su participación. En cuanto al número de hijos, contrario a las afirmaciones de Lehman et al. (1999: 51), la preocupación por la educación y salud de los mismos actúan en forma de incentivos al activismo político tanto de hombres como de mujeres.

10 Se construyó una dummy de divorcio con el fin exclusivo de comprobar la teoría de Iversen y Rosenbluth (2006).

11 Los resultados presentados son robustos a la medición de participación política: como el promedio simple o ponderado. 
Recordemos que la ideología explícita se mide a partir del tipo de ideología que los individuos reportan tener, mientras que la implícita se construye a partir de un promedio aritmético del nivel de acuerdo de los individuos frente a diversas afirmaciones que presuponen un tipo de ideología. Un mayor valor en el índice de ideología implícita está asociado con un aumento en la participación tanto para hombres como para mujeres. Esto implica que en términos de ideología implícita el ser más de izquierda aumenta la probabilidad de participación. Este hallazgo es consistente con las afirmaciones de Iversen y Rosenbluth (2006: 12), quienes encuentran que las mujeres con una ideología de izquierda son más activas políticamente. La ideología explícita, en cambio, no resulta significativa en ninguna de las especificaciones, lo cual es sorprendente. Como se mostró arriba, no existe mucha consistencia entre las ideologías explícita e implícita. Posiblemente se puede atribuir a las diferencias en prospectos políticos en el continente, en donde los individuos tienen diferentes umbrales para considerarse de izquierda o derecha. Esto podría explicar la falta de significancia de la ideología explícita.

La ocupación aparece como un determinante relevante de la participación política femenina, mientras que no es estadísticamente significativa para la masculina. En general, a mayor proporción de mujeres participando activamente en el mercado laboral, mayor su participación política. Esto puede interpretarse como un efecto de empoderamiento que tiene el participar en el mercado laboral sobre las mujeres, volviéndolas más interesadas en política y en la toma de decisiones. También puede ser que las mujeres que trabajan fuera del hogar se dan cuenta de más instancias en las que son discriminadas, por ejemplo vía menores salarios, y esto las hace interesarse más en la política. Este hallazgo apoya la teoría de Lehman et al. (1999: 30) y la de Iversen y Rosenbluth (2006: 17), quienes encuentran que al ocuparse del hogar, las mujeres se aíslan del ámbito público y por tanto su participación política es menor.

El ingreso es otro ejemplo de una variable que determina la participación política de las mujeres mas no la de los hombres. Al tener mayores ingresos, y por ende pagar mayores impuestos al Estado, el interés por el tipo de políticas públicas que se llevan a cabo aumenta, y por tanto las mujeres son más activas políticamente.

Por su parte, la confianza en hombres líderes resulta ser un determinante significativo de la participación política masculina mas no de la femenina. Históricamente, es más común que haya hombres líderes políticos que mujeres. Esto genera más referentes de líderes políticos hombres; en el caso de las mujeres, esos referentes son muy aislados.

La variable de zona urbana, medida por una dummy que toma el valor de 1 si el individuo habita en una zona urbana, disminuye la participación política de los hombres y no afecta la de las mujeres. Esto implica que las mujeres urbanas y rurales participan por igual, mientras que los hombres rurales tienen mayores niveles de participación. Esto podría deberse a la dependencia del Estado de las zonas rurales.

Finalmente se observa el comportamiento del estado civil, medido por una dummy de divorcio. La variable de estado civil no resulta significativa para la participación política masculina pero sí tiene un efecto negativo y estadísticamente significativo para las mujeres. Contrario a lo establecido por Iversen y Rosenbluth (2006:18), los resultados 
sugieren que las mujeres divorciadas suelen participar menos que las solteras, casadas, viudas o en unión marital de hecho. En el Anexo se presentan los resultados de los efectos fijos por país para la estimación principal, teniendo a Colombia como país excluido.

A manera de robustez, decidimos repetir la estimación combinando los índices de ideología explícita e implícita, haciendo un promedio simple de los dos. Los resultados, que se reportan en el Anexo, son bastante similares en términos de coeficientes y significancia estadística a los obtenidos en la estimación principal en términos de las demás variables de control. El índice compuesto de ideología afecta positivamente la participación política de los hombres, pero no resulta significativo para las mujeres.

\section{CONCLUSIONES}

Existen diferencias entre los determinantes de la participación política de mujeres y hombres en América Latina. Las estimaciones del modelo sugieren que la ocupación, el nivel de ingreso y el estado civil están asociados con el activismo político de las mujeres en América Latina. Por su parte, la confianza en hombres como líderes políticos y vivir en una zona urbana afectan la participación política masculina.

Las mujeres divorciadas son menos activas políticamente que las demás mujeres. Dado que este resultado difiere de lo esperado en base a la literatura, se sugiere explorar a mayor profundidad la potencial relación causal tras esta relación.

Como la participación femenina en el mercado laboral está asociada con un aumento en la participación política, dado que la participación femenina ha aumentado en el mercado laboral en las últimas décadas en la región, es posible que esto se traduzca en un aumento en la participación política femenina.

Todavía queda bastante por investigar. Por ejemplo, la participación ciudadana es aún un tema poco explorado en la región, más aún en términos de género. Futuros artículos podrían explorar las diferencias entre países de Latinoamérica, entendiendo qué puede explicar las diferencias de participación entre países. También debería verificarse la validez de las relaciones causales planteadas, principalmente en los resultados contra intuitivos, como lo es la relación negativa con el divorcio. De igual forma, sería deseable repetir el análisis usando datos de tipo panel. ${ }^{12}$

\section{REFERENCIAS}

Abeyasekera, Savitri. 2005. “Multivariate Methods for Index Construction". En Household Surveys in Developing and Transition Countries: Design, Implementation and Analysis, Series F N ${ }^{\circ}$ 96, Nueva York: Naciones Unidas Editores [En línea] http://unstats.un.org/unsd/hhsurveys/finalpublication/ch18fin3.pdf

12 No fue posible la creación de un panel de datos, debido a que los individuos cambian en el tiempo en la base de datos de LAPOP. Una aproximación potencial a este problema podría darse, a partir de la creación de un panel simulado, coincidiendo las características de grupos de individuos. 
AECID Embajada de España en Colombia. 2007. Situación de las Mujeres en Colombia y Avances en Políticas de Igualdad. Bogotá: AECID.

Altman, David. 2009. "Individual, Economic, and Institutional Causes of Electoral Participation in Latin America". Instituto de Ciencia Política de la Universidad Católica de Chile. [En línea] http:/ / sitemason. vanderbilt.edu/files/heU6Y0/ALTMAN\%20Individual\%20Economic\%20and\%20Institutional\%20 Causes\%20of\%20Electoral\%20Participation\%20in\%20Latin\%20America.pdf, [Consulta: 22-2-2010].

Barnes, Samuel y Max Kaase. 1979. Political Action: Masss Participation in Five Western Democracies. Beverly Hills: Sage University Press.

Biblioteca Luis Ángel Arango. 2005. "Guía temática de política”. [En línea] http:/ /www.lablaa.org/ blaavirtual/ayudadetareas/poli/poli89.htm [Consulta: 16-2-2010].

Booth, John y Mitchell Seligson. 1976. "Political Participation in Latin America: An Agenda for Research". Latin American Research Review 11: 95-119.

Booth, John y Mitchell Seligson. 1978. Political Participation in Latin America. New York: Holmes and Meier.

Centro Nacional de Consultoría, y Corporación Humanas Colombia. 2009. Encuesta Nacional de Mujeres: Interés de las Mujeres en la Política e Intención de Voto. Bogotá, Colombia: Humanas Colombia.

Cole, Elizabeth, Alyssa Zucker y Joan Ostrove. 1998. "Political Participation and Feminist Consciousness among Women Activists". Political Psychology 19 (2): 349-71.

Conge, Patrick. 1988. "The Concept of Political Participation: Toward a Definition". Comparative Politics 20 (2): 241-49.

Cordell, Matthew. 2010. "Women on the Rise in South American Politics". UN dispatch Global News and Views. [En línea] http://www.undispatch.com/women-on-the-rise-in-south-american-politics [Consulta: 10-2-2011].

Donoso, Alina y Teresa Valdés. 2007. Participación política de las mujeres en América Latina. Informe regional. Chile: Centro de Estudios para el Desarrollo de la Mujer CEDEM.

Donoso, Alina y Teresa Valdés. 2007. Participación política de las mujeres en América Latina. Chile: ALOP Asociación Latinoamericana de Organizaciones de Promoción al Desarrollo.

Duque, Sylvia. 2008. De qué manera el control territorial paramilitar modifica la participación política. Bogotá: Trabajo de investigación para optar al título de politóloga, Universidad de los Andes.

FLACSO. 2006. "Mujeres Latinoamericanas en Cifras". [En línea] http://www.eurosur.org/FLACSO/ mujeres/ [Consulta: 22-2-2010].

Inglehart, Ronald y Pippa Norris. 2003. The Gender Gap in Voting and Public Opinion, Cambridge: Cambridge University Press.

Inglehart, Ronald, Pippa Norris y Christian Welzel. 2004. "Gender Equality and Democracy". [En línea] http://www.fd.unl.pt/docentes_docs/ma/tpb_MA_6187.pdf [Consulta: 20-10-2010].

Injuve. 2006. "Jóvenes, derechos y ciudadanía - Instituto de la Juventud". [En línea] http:/ /www.injuve. migualdad.es/injuve/contenidos.downloadatt.action?id=1352706234 [Consulta: 20-9-2010].

Iversen, Torben y Frances Rosenbluth. 2006. “The Political Economy of Gender: Explaining Cross National Variation in the Gender Division of Labor and Gender Voting Gap". American Journal of Political Science 50 (1): 1-28.

Llanos, Beatriz y Kristen Sample. 2008. 30 años de democracia: ¿en la cresta de la ola? Participación Política de la mujer en América Latina. Estocolmo: International Institute for Democracy and Electoral Assistance.

Milbrath, Lester. 1965. Political Participation: How and Why Do People Get Envolved in Politics? Chicago: Rand McNally.

Milosavljevic, Vivian. 2007. Estadísticas para la equidad de género: magnitudes y tendencia en América Latina. Santiago de Chile: CEPAL.

Morales Diez, Laura. 1998. Participación Política y pertenencia a grupos políticos: los límites de las explicaciones individuales y la necesidad de considerar el contexto político. Madrid: Reis.

Peschard, Jacqueline. 2002. "El sistema general de cuotas en América Latina: Panorama general". En Mujeres en el Parlamento: Más allá de los números. [En línea] http:/ /www.idea.int/publications/wip/ upload/chapter_04a-CS-LatinAmerica.pdf [Consulta: 16-2-2010].

PNUD. 2004. La democracia en América Latina: Hacia una democracia de ciudadanas y ciudadanos. New York: Programa de las Naciones Unidas para el Desarrollo. 
Prado, Mario Fernando. 2010. “Por qué mujer no vota mujer?” El Espectador. [En línea] http://www. elespectador.com/columna187206-mujer-no-vota-mujer [Consulta: 16-2-2011].

Ranaboldo, Claudia y Yolanda Solana. 2008. Desigualdad de género en la participación política de las mujeres en América Latina y el Caribe. Santiago de Chile: Programa de Dinámicas Territoriales Rurales Rimisp.

Sabucedo, Manuel. 1986. Psicología política: Factores explicativos de la participación electoral. Madrid: Revista de Investigación Psicológica.

Schlozman, Lehman, Nancy Burns y Sidney Verba. 1999. “What happened at work today: A Multistage Model of Gender, Employment and Political Participation". The Journal of Politics 61 (1): 29-53.

Sanbonmatsu, Kira. 2002. "Gender Stereotypes and Vote Choice". American Journal of Political Science 46 (1): 20-34.

Sanbonmatsu, Kira. 2003. "Gender-Related Political Knowledge and the Descriptive Representation of Women". Political Behavior 25 (4): 367-88.

Turbay, María M. 2005. Una mirada de género a la encuesta de calidad de vida 2003: Jefatura del hogar y seguridad social en Colombia. Bogotá: Cuadernos PNUD - Consejería Presidencial para la Equidad de la Mujer.

Verba, Sydney. 1967. "Democratic Participation". Annals of the American Academy of Political and Social Science 373 (1): 53-78.

Wills, María E. 2005. “¿Por qué incluir no es igual a representar? La trayectoria de las mujeres hacia la política en Colombia 1954-2003". Seminario IEPRI, Universidad Nacional de Colombia, Bogotá, noviembre 2005.

Wills, María E. 2007. “Las dinámicas de la representación política de las mujeres y su impacto en la construcción de ciudadanía en Colombia 1974-2000: las coincidencias desafortunadas". Seminario Internacional: Identidades, movilizaciones sociales y politica en América Latina, Universidad de los Andes, Bogotá, 6-8 noviembre 2007.

Zechmeister, Elizabeth. 2006. "What's Left and Who's Right? A Q-Method Study of Individual and Contextual Influences on the Meaning of Ideological Labels". Political Behavior 28 (2): 151-73.

Zimpel, Gisela. 1980. "Participación política". En Diccionario de Ciencia Política, editado por F. Ehrenwirth. Madrid: Alianza Editorial, pp. 473-78. 


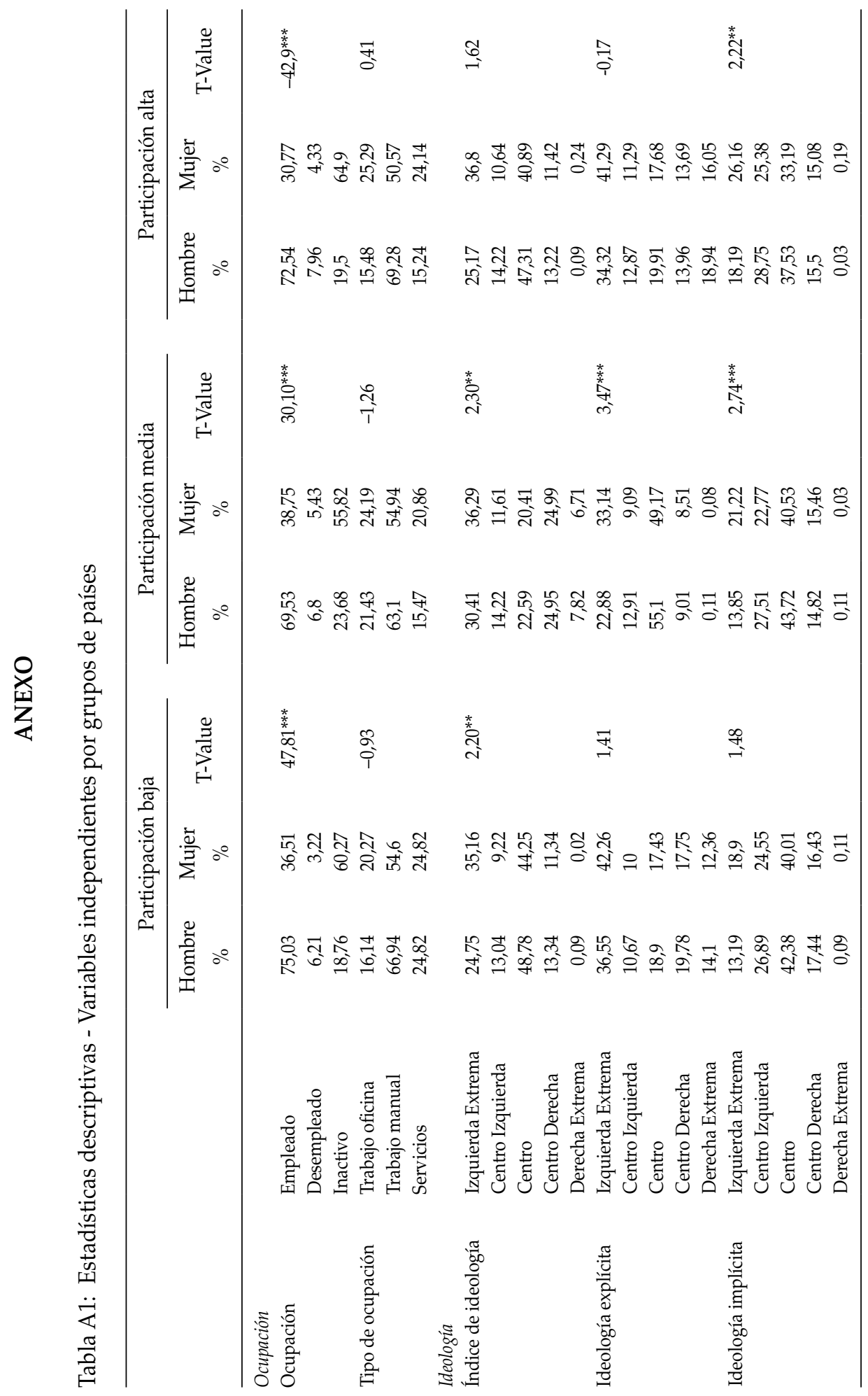




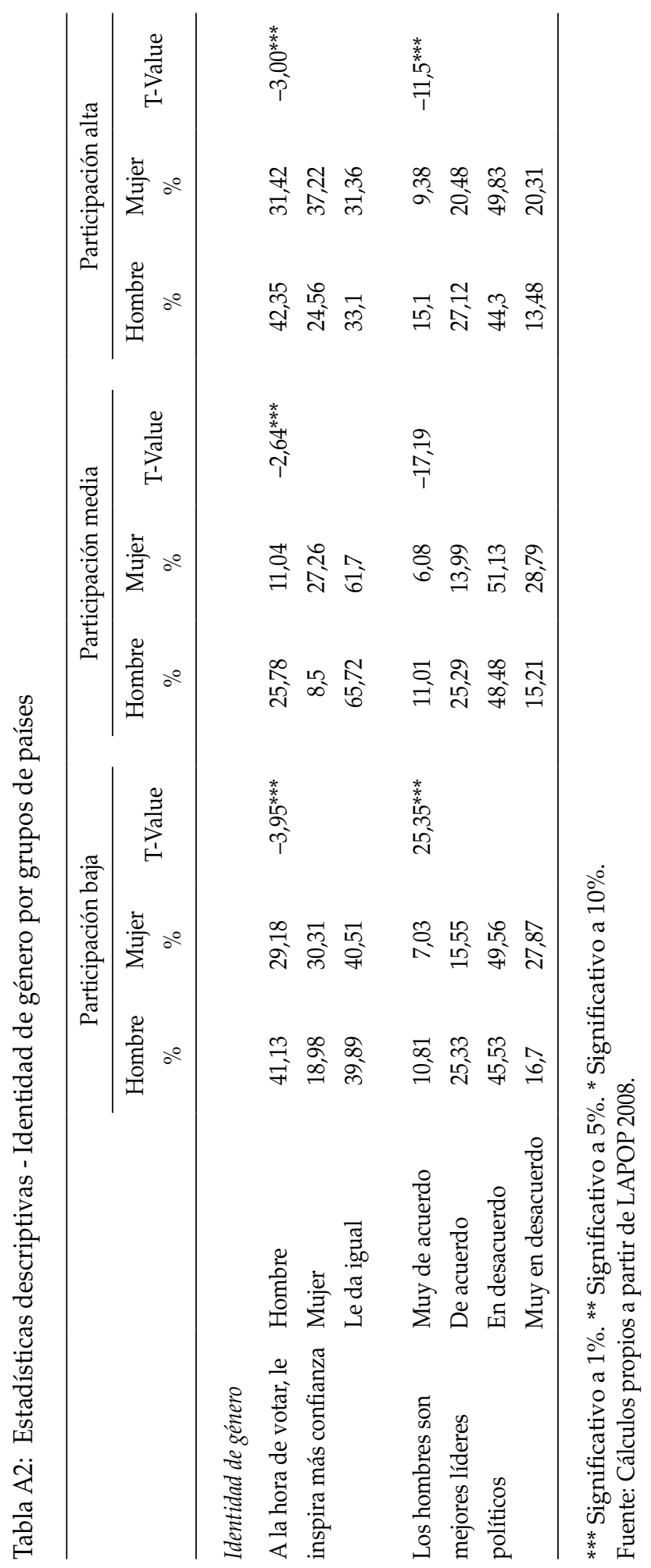


Tabla A3: Probabilidad de participación - Efectos fijos por país

\begin{tabular}{|c|c|c|c|c|}
\hline & $\begin{array}{c}(1) \\
\text { Mujeres }\end{array}$ & $\begin{array}{c}(2) \\
\text { Hombres }\end{array}$ & $\begin{array}{c}(3) \\
\text { Mujeres }\end{array}$ & $\begin{array}{c}(4) \\
\text { Hombres }\end{array}$ \\
\hline México & $\begin{array}{c}0,017 \\
(0,073)\end{array}$ & $\begin{array}{l}-0,275^{* * *} \\
(0,074)\end{array}$ & $\begin{array}{c}0,011 \\
(0,073)\end{array}$ & $\begin{array}{l}-0,280^{* * *} \\
(0,074)\end{array}$ \\
\hline Guatemala & $\begin{array}{l}-0,030 \\
(0,079)\end{array}$ & $\begin{array}{l}-0,215^{* * *} \\
(0,082)\end{array}$ & $\begin{array}{c}-0,041 \\
(0,080)\end{array}$ & $\begin{array}{l}-0,229^{* * *} \\
(0,082)\end{array}$ \\
\hline El Salvador & $\begin{array}{l}0,137^{* *} \\
(0,068)\end{array}$ & $\begin{array}{c}-0,021 \\
(0,072)\end{array}$ & $\begin{array}{l}0,133^{* *} \\
(0,067)\end{array}$ & $\begin{array}{l}-0,031 \\
(0,072)\end{array}$ \\
\hline Honduras & $\begin{array}{l}0,361^{* * *} \\
(0,079)\end{array}$ & $\begin{array}{l}0,284^{* * *} \\
(0,080)\end{array}$ & $\begin{array}{l}0,335^{* * *} \\
(0,078)\end{array}$ & $\begin{array}{l}0,263^{* * *} \\
(0,080)\end{array}$ \\
\hline Nicaragua & $\begin{array}{l}0,405^{* * *} \\
(0,078)\end{array}$ & $\begin{array}{l}0,449^{* * *} \\
(0,078)\end{array}$ & $\begin{array}{l}0,401^{* * *} \\
(0,077)\end{array}$ & $\begin{array}{l}0,448^{* * * *} \\
(0,078)\end{array}$ \\
\hline Costa Rica & $\begin{array}{c}0,113 \\
(0,077)\end{array}$ & $\begin{array}{c}-0,074 \\
(0,077)\end{array}$ & $\begin{array}{c}0,111 \\
(0,077)\end{array}$ & $\begin{array}{l}-0,075 \\
(0,077)\end{array}$ \\
\hline Panamá & $\begin{array}{c}0,109 \\
(0,072)\end{array}$ & $\begin{array}{c}-0,063 \\
(0,075)\end{array}$ & $\begin{array}{c}0,101 \\
(0,072)\end{array}$ & $\begin{array}{l}-0,072 \\
(0,075)\end{array}$ \\
\hline Ecuador & $\begin{array}{c}0,074 \\
(0,067)\end{array}$ & $\begin{array}{c}-0,097 \\
(0,068)\end{array}$ & $\begin{array}{c}0,059 \\
(0,067)\end{array}$ & $\begin{array}{c}-0,111 \\
(0,068)\end{array}$ \\
\hline Bolivia & $\begin{array}{l}0,204^{* * *} \\
(0,067)\end{array}$ & $\begin{array}{c}0,131^{*} \\
(0,068)\end{array}$ & $\begin{array}{l}0,200^{* * *} \\
(0,067)\end{array}$ & $\begin{array}{c}0,126^{*} \\
(0,068)\end{array}$ \\
\hline Perú & $\begin{array}{c}0,052 \\
(0,071)\end{array}$ & $\begin{array}{c}-0,149^{* *} \\
(0,073)\end{array}$ & $\begin{array}{c}0,046 \\
(0,071)\end{array}$ & $\begin{array}{c}-0,154^{* *} \\
(0,073)\end{array}$ \\
\hline Paraguay & $\begin{array}{l}0,536^{* * *} \\
(0,088)\end{array}$ & $\begin{array}{l}0,417^{* * *} \\
(0,085)\end{array}$ & $\begin{array}{l}0,538^{* * *} \\
(0,088)\end{array}$ & $\begin{array}{l}0,420^{* * *} \\
(0,085)\end{array}$ \\
\hline Uruguay & $\begin{array}{l}0,484^{* * *} \\
(0,070)\end{array}$ & $\begin{array}{l}0,371^{* * *} \\
(0,075)\end{array}$ & $\begin{array}{l}0,478^{* * *} \\
(0,071)\end{array}$ & $\begin{array}{l}0,362^{* * *} \\
(0,075)\end{array}$ \\
\hline Brasil & $\begin{array}{l}0,249^{* * *} \\
(0,077)\end{array}$ & $\begin{array}{c}0,157^{*} \\
(0,082)\end{array}$ & $\begin{array}{l}0,234^{* * *} \\
(0,077)\end{array}$ & $\begin{array}{c}0,143^{*} \\
(0,082)\end{array}$ \\
\hline R. Dominicana & $0,752^{* * *}$ & $0,595^{* * *}$ & $0,763^{* * *}$ & $0,604^{* * *}$ \\
\hline $\mathrm{N}$ & 15.023 & 15.964 & 15.023 & 15.964 \\
\hline $\mathrm{R}^{2}$ & 0,099 & 0,114 & 0,098 & 0,113 \\
\hline
\end{tabular}

Errores estándares robustos en paréntesis. Efectos fijos por país; país excluido Colombia. *** Significativo a $1 \%$. ${ }^{* *}$ Significativo a $5 \%$. ${ }^{*}$ Significativo a $10 \%$.

Fuente: Realizado por autoras en base a LAPOP, 2008. 
Tabla A4: Estimación con índice de ideologías por promedio simple

\begin{tabular}{|c|c|c|c|c|}
\hline & $\begin{array}{c}\text { (1) } \\
\text { Mujeres }\end{array}$ & $\begin{array}{c}(2) \\
\text { Hombres }\end{array}$ & $\begin{array}{c}(3) \\
\text { Mujeres }\end{array}$ & $\begin{array}{c}(4) \\
\text { Hombres }\end{array}$ \\
\hline Ocupación & $\begin{array}{l}0,198^{* * *} \\
(0,028)\end{array}$ & $\begin{array}{c}0,320 \\
(0,032)\end{array}$ & $\begin{array}{l}0,196^{* * *} \\
(0,028)\end{array}$ & $\begin{array}{c}0,323 \\
(0,032)\end{array}$ \\
\hline Ideología explícita & $\begin{array}{c}0,001 \\
(0,005)\end{array}$ & $\begin{array}{c}0,008 \\
(0,005)\end{array}$ & & \\
\hline Ideología implícita & $\begin{array}{l}0,069^{* * *} \\
(0,018)\end{array}$ & $\begin{array}{l}0,087^{* * *} \\
(0,018)\end{array}$ & & \\
\hline Confianza en hombres líderes & $\begin{array}{c}0,009 \\
(0,016)\end{array}$ & $\begin{array}{l}0,072^{* * *} \\
(0,015)\end{array}$ & $\begin{array}{c}0,010 \\
(0,016)\end{array}$ & $\begin{array}{l}0,072^{* * *} \\
(0,015)\end{array}$ \\
\hline Educación & $\begin{array}{l}0,134^{* * *} \\
(0,012)\end{array}$ & $\begin{array}{l}0,157^{* * *} \\
(0,012)\end{array}$ & $\begin{array}{l}0,133^{* * *} \\
(0,012)\end{array}$ & $\begin{array}{l}0,155^{* * *} \\
(0,012)\end{array}$ \\
\hline Urbano/Rural & $\begin{array}{l}-0,018 \\
(0,031)\end{array}$ & $\begin{array}{l}-0,110^{* * *} \\
(0,031)\end{array}$ & $\begin{array}{r}-0,0170 \\
(0,031)\end{array}$ & $\begin{array}{l}-0,110^{* * *} \\
(0,031)\end{array}$ \\
\hline Edad & $\begin{array}{l}0,012^{* * *} \\
(0,001)\end{array}$ & $\begin{array}{l}0,014^{* * *} \\
(0,001)\end{array}$ & $\begin{array}{l}0,011^{* * *} \\
(0,001)\end{array}$ & $\begin{array}{l}0,014^{* * *} \\
(0,001)\end{array}$ \\
\hline Estado civil & $\begin{array}{l}-0,114^{* * *} \\
(0,033)\end{array}$ & $\begin{array}{l}-0,098 \\
(0,036)\end{array}$ & $\begin{array}{l}-0,112^{* * *} \\
(0,033)\end{array}$ & $\begin{array}{l}-0,097 \\
(0,036)\end{array}$ \\
\hline Ingreso & $\begin{array}{l}0,016^{* *} \\
(0,007)\end{array}$ & $\begin{array}{c}0,011 \\
(0,007)\end{array}$ & $\begin{array}{l}0,015^{* *} \\
(0,007)\end{array}$ & $\begin{array}{c}0,009 \\
(0,007)\end{array}$ \\
\hline Número de hijos & $\begin{array}{l}0,041^{* * *} \\
(0,008)\end{array}$ & $\begin{array}{l}0,038^{* * *} \\
(0,008)\end{array}$ & $\begin{array}{l}0,043^{* * *} \\
(0,008)\end{array}$ & $\begin{array}{l}0,039^{* * *} \\
(0,008)\end{array}$ \\
\hline Índice de ideologías & & & $\begin{array}{c}0,014 \\
(0,010)\end{array}$ & $\begin{array}{l}0,031^{* * *} \\
(0,010)\end{array}$ \\
\hline Constante & $\begin{array}{l}-1,563^{* * *} \\
(0,124)\end{array}$ & $\begin{array}{c}-1,783^{* * *} \\
(0,133)\end{array}$ & $\begin{array}{l}-1,232^{* * *} \\
(0,086)\end{array}$ & $\begin{array}{c}-1,332^{* * *} \\
(0,098)\end{array}$ \\
\hline $\mathrm{N}$ & 15.023 & 15.964 & 15.023 & 15.964 \\
\hline $\mathrm{R}^{2}$ & 0,099 & 0,114 & 0,098 & 0,113 \\
\hline
\end{tabular}

Errores estándares robustos en paréntesis.

${ }^{* * *} \mathrm{p}<1 \%$, ${ }^{* *} \mathrm{p}<5 \%,{ }^{*} \mathrm{p}<10 \%$.

Fuente: Realizado por autoras en base a LAPOP, 2008.

Mónica Pachón. Profesora asociada en Ciencia Política de la Universidad de los Andes, Colombia. Ph.D. en Ciencia Política, Universidad de California, San Diego y M.Phil. Estudios Latinoamericanos, Universidad de Oxford.

E-mail: mopachon@uniandes.edu.co

Ximena Peña. Profesora asistente en Economía de la Universidad de los Andes, Colombia. Ph.D. en Economía, Georgetown University.

E-mail: xpena@uniandes.edu.co

Mónica Wills. Asesora en la Fundación Ideas para la Paz. Economista de la Universidad de los Andes y politóloga con concentración en política colombiana y política comparada de la misma universidad. E-mail: willsmonica@gmail.com. 
